\title{
Die DGAI feiert 50-jähriges Bestehen
}

U nter dem Eindruck es wissenschaftliches Forum für allergologische Fragestellungen zu schaffen, wurde vor 50 Jahren, am 17. Juni 1951, die DGAI in Frankfurt am Main gegründet. So schrieb dem Erstunterzeichner am 9. Juli 2001 das Gründungsmitglied Prof. Dr. Dr. h.c. Hans Erhard Bock, Tübingen, im 98. Lebensjahr. Damit gelang gleichzeitig ein wichtiger Schritt im Nachkriegsdeutschland - die Kontaktaufnahme zur internationalen Allergologie, insbeson-
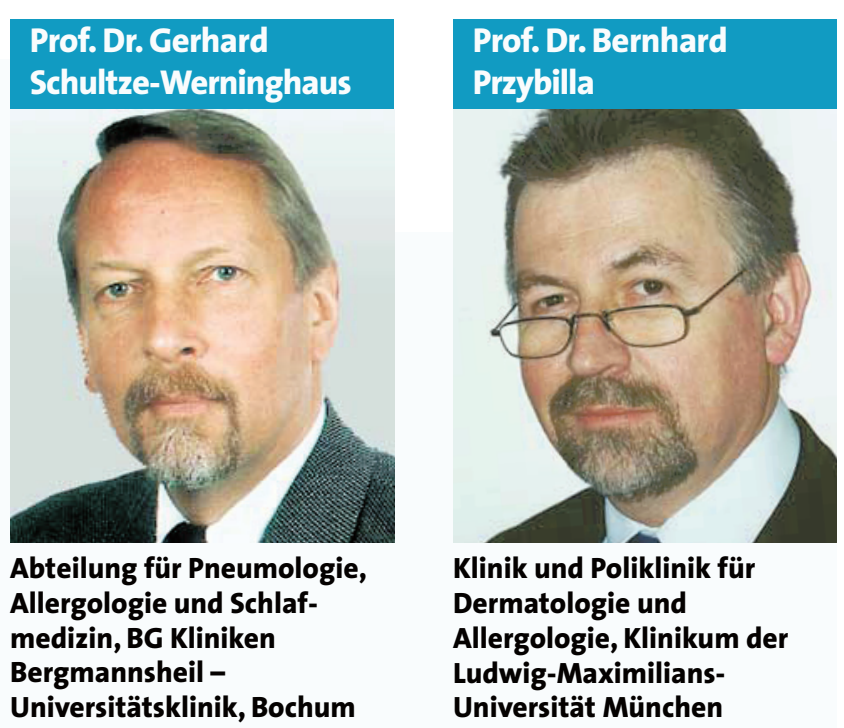
sei an der Zeit, ein

Josef Wenning, die auf verschiedensten Ebenen wissenschaftspolitisch vieles eingeleitet und umgesetzt haben. Dazu gehört die wesentliche Verbesserung der Öffentlichkeitsarbeit, vor allem durch die Verpflichtung der Agentur MasterMedia, die Einrichtung von Geschäftsstellen beider Verbände, die zunehmende Zahl von Fortbildungsveranstaltungen mit Unterstützung der Verbände und der neugegründeten Deutschen Akademie für Allergologie und Umweltmedizin (DAAU), die Zusammenardere gefördert von Karl Hansen. Im gleichen Jahr wurde in Zürich der erste internationale Allergie-Kongress veranstaltet, geleitet von dem Zürcher Internisten Wilhelm Loeffler, der 1932 das Krankheitsbild des seinen Namen tragenden eosinophilen Lun„DGAl und ÅDA haben geninfiltrats bein den letzten Jahren an schrieb. Im Jahre vielen Brennpunkten 2005 wird dieser Akzente gesetzt" Kongress unter der Präsidentschaft von Johannes Ring in München zum 19. Male statt finden, Zeichen dafür, dass diese internationalen Kontakte Früchte getragen haben (Ein ausführlicher Beitrag zur Gründungsgeschichte folgt in der Ausgabe 8/01).

Die Entwicklung der Allergologie ist gekennzeichnet von einem wissenschaftlich belegten Anstieg allergischer Erkrankungen. Auch die Zunahme des öffentlichen Bewusstseins und Interesses an den auslösenden Faktoren machen Allergien und Umwelt zu den wichtigsten medizinischen Themen in den Medien. Demgegenüber stehen Defizite in der Aus- und Weiterbildung, der Patienten-Versorgung in Praxis und Klinik, in Strukturen und Forschungsförderung. Diese Defizite resultieren in einer Unter- und Fehlversorgung, aber auch Überversorgung der Allergiker. Einerseits werden zahlreiche Allergie-Tests in vivo und in vitro durchgeführt, aus denen keine erkennbaren Konsequenzen resultieren, was den Medizin-Tourismus in unsinniger Weise fördert, zum anderen werden Chancen zur Prävention, Frühdiagnostik und -therapie nicht hinreichend genutzt, die mögliche Verhinderung eines Etagenwechsels versäumt.

Bessere Konzepte für die allergologische Versorgung müssen zum einen die verantwortlichen politischen und standespolitischen Institutionen in Kooperation mit den Fachverbänden schaffen. Aber zum anderen kann sie jeder von uns fördern durch Vermeidung von überflüssigen In-vivo- und In-vitro-Testungen, fehlerhaften Indikationstellungen bzw. unbegründeter Ablehnung der spezifischen Immuntherapie und inadäquater Pharmakotherapie.

DGAI und ÄDA haben in den letzten Jahren an vielen Brennpunkten Akzente gesetzt. Besonders zu nennen sind dabei die Namen Johannes Ring und

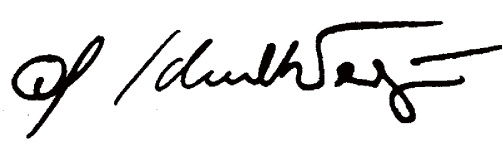

Prof. Dr. G. Schultze-Werninghaus beit mit der politischen Ebene wie im Aktionsbündnis Allergieprävention (ABAP) und vor allem die Veröffentlichung des Weißbuches Allergie 2000.

Allergische Erkrankungen sind Volkskrankheiten wie Diabetes mellitus, koronare Herzerkrankung, Bluthochdruck, schlafbezogene Atmungsstörungen und Krebs. Daher ist jeder von uns aufge- „Allergien sind fordert, durch eigene Volkskrankheiten" Aktivitäten zu der erfor-

derlichen Präsenz der Allergologie auf allen Ebenen beizutragen. Ein wichtiger Markstein wird auch die kommende 22. Tagung der DGAI zusammen mit der Deutschen Gesellschaft für Pneumologie (DGP) vom 13.-16. März 2002 in Bochum sein. Um Anmeldungen von Postern und Kurzvorträgen zu allen Themen der Allergologie bis zum 10. September 2001 wird gebeten: http://www.bochum2002.de

\section{Mit kollegialen Grüßen,}

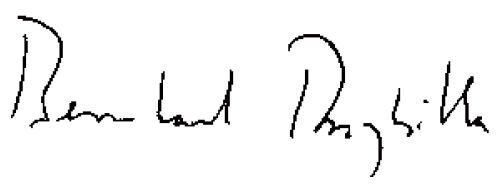

Prof. Dr. B. Przybilla 